Advances in Fuzzy Mathematics.

ISSN 0973-533X Volume 12, Number 3 (2017), pp. 549-575

(C) Research India Publications

https://dx.doi.org/10.37622/AFM/12.3.2017.549-575

\title{
Multi-Objective Welded Beam Design Optimization using T-Norm and T-Co-norm based Intuitionistic Fuzzy Optimization Technique
}

\author{
Mridula Sarkar* \\ Department of Mathematics, Indian Institute of Engineering Science and Technology, \\ Shibpur, P.O.-Botanic Garden, Howrah-711103, West Bengal, India. \\ *corresponding author \\ Tapan Kumar Roy \\ Department of Mathematics, Indian Institute of Engineering Science and Technology, \\ Shibpur, P.O.-Botanic Garden, Howrah-711103, West Bengal, India.
}

\begin{abstract}
Real world engineering problems are usually designed by the presence of many conflicting objectives. In this paper we develop an approach to solve multi-objective welded beam design using t-norms and t- co-norms based intuitionistic fuzzy optimization technique. Here binary t-norms, $t$-co-norms are extended in the form of n-ary t-norms and t-co-norms and their basic properties are discussed with some special cases. In this paper we have considered optimization problem of minimization of the cost of welding and deflection at the tip of a welded steel beam, while the maximum shear stress in the weld group, maximum bending stress in the beam, and buckling load of the beam have been considered as constraints. The problem of designing an optimal welded beam consists of dimensioning a welded steel beam and the welding length so as to minimize its cost, subject to the constraints as stated above. This classical welded beam optimization example is presented here in to demonstrate the efficiency of our proposed optimization approach. Numerical example is given here to illustrate this structural model through this approximation method.
\end{abstract}

Keyword: Intuitionistic Fuzzy Set, t-norms,t-co-norms, Multi-Objective Intuitionistic Optimization, Welded Beam Optimization. 


\section{INTRODUCTION}

Optimization is a technique that deal with the problem of minimizing or maximizing a certain function over a subset in a finite dimensional Euclidean space, which is determined by functional inequalities .It has been seen that in numerous engineering design problem objectives under consideration conflict with each other, and optimizing the problem considering a single objective can result an unacceptable results with respect to the other objectives. A reasonable solution to a multi-objective problem a set of solutions, each of which satisfies the objectives without being dominated by any other solution.There are two general approaches for multipleobjective optimization. One of this is , make individual objective functions by combining all objective functions into a single composite function or move all objective but one to the constraint set. In the former case, determination of a single objective can be made by utility theory or weighted sum method, where weights or utility functions are dependent on the decision-maker's preferences. Sometimes, it can be very difficult to accurately select these weights. In the latter case, as a constraining value must be established for each of these former objectives there is a problem to move objectives to the constraint set. Again this can be arbitrary.So in both cases, a set of solutions in exchange of single solution would return by optimization method for examination of trade-offs. For this reason, decision-makers often choose a set of good solutions considering the multiple objectives. Welding, a process of joining metallic parts with the application of heat or pressure or the both, with or without added material, is an economical and efficient method for obtaining permanent joints in the metallic parts. This welded joints are generally used as a substitute for riveted joint or can be used as an alternative method for casting or forging. The welding processes can broadly be classified into following two groups, the welding process that uses heat alone to join two metallic parts and the welding process that uses a combination of heat and pressure for joining (Bhandari. V. B). However, above all the design of welded beam should preferably be economical and durable one. Since decades, deterministic optimization has been widely used in practice for optimizing welded connection design. These include mathematical traditional optimization algorithms (Ragsdell \& Phillips [1]) ,GA-based methods (Deb [2], Deb [3], Coello [4], Coello [5]), particle swarm optimization (Reddy [6]), harmony search method (Lee \& Geem [7]), and Big-Bang Big-Crunch (BB-BC) algorithm (O. Hasançebi, [8]), subset simulation (Li [9]), improved harmony search algorithm (Mahadavi [10]), were other methods used to solve this problem. All these deterministic optimizations aim to search the optimum solution under given constraints without consideration of uncertainties. So, while a deterministic optimization approach is unable to handle structural performances such as imprecise stresses and deflection etc. due to the presence of impreciseness, to get rid of such problem fuzzy (Zadeh, [11]), intuitionistic fuzzy (Atanassov,[12]) play great roles.

It has been seen that numerous engineering design problem need to deal with noisy data, manufacturing error or uncertainty of the environment during the design process. Fuzzy as well as intuitionistic fuzzy optimization in case of structural engineering play great role in their design and analysis and optimize the model. This fuzzy set 
theory was first introduced by Zadeh(1965). As an extension Intuitionistic fuzzy set theory was first introduced by Attanassov(1986). When an imprecise information can not be expressed by means of conventional fuzzy set Intuitionistic Fuzzy set play an important role. In intuitionistic fuzzy (IF) set we usually consider degree of acceptance, degree of non membership and a hesitancy function whereas we consider only membership function in fuzzy set. A few research work has been done on intuitionistic fuzzy optimization in the field of structural optimization. Dey et al.[14]used t-norm and t-conorm based intuitionistic fuzzy technique to optimize multi objective truss structural model.

An important concept in fuzzy as well as intuitionistic fuzzy set theory is triangular norms and conforms which are nothing but a generalized intersection and union of fuzzy sets. Alsina et.al [15] introduced the t-norm in fuzzy set theory and suggested that the t-norms

could be used for the intersection of fuzzy sets .G. Deschrijver et. al [16] introduced the concept of intuitionistic fuzzy t-norm and t-co norm to investigate the theorems for similar representation of aggregated t-norm and t-conorm.

As per our best of knowledge this is the first time basic t-norms and t- co-norm based intuitionistic fuzzy optimization programming technique is being used to solve multi-objective welded beam design in this paper. In the test problem is of minimization of the cost of welding and deflection at the tip of a welded steel beam, while the maximum shear stress in the weld group, maximum bending stress in the beam, and buckling load of the beam have been considered as constraints. The remainder of this paper is organized in the following way. In section 2 structural optimization model is discussed. In section 3, mathematics Prerequisites are discussed with . extended n-ary t-norms and t-co-norms and calculation of some of special cases. In section 4, we discuss about weighted fuzzy aggregation In section 5, we proposed the technique to solve a multi-objective non-linear programming problem using extended t-norms and t-co-norm based intuitionistic fuzzy optimization. In section 6, multi-objective structural model is solved using extended t-norms and t-co-norm based intuitionistic fuzzy optimization. Numerical illustration of structural model of welded beam design and comparison of results by using different extended weighted t-norms and t-co-norm are discussed in section 7.Finally we draw conclusions in section 8 .

\section{MULTI-OBJECTIVE STRUCTURAL MODEL}

In sizing optimization problems, the aim is to minimize multi objective function, usually the cost of the structure, deflection under certain behavioural constraints which are displacement or stresses. The design variables are most frequently chosen to be dimensions of the height, length, depth and width of the structures. Due to fabrications limitations the design variables are not continuous but discrete for 
belongingness of cross-sections to a certain set. A discrete structural optimization problem can be formulated in the following form

Minimize $C(X)$

Minimize $\delta(X)$

subject to $\sigma_{i}(X) \leq\left[\sigma_{i}\right], i=1,2, \ldots ., m$

$X_{j} \in R^{d}, \quad j=1,2, \ldots . ., n$

where $C(X), \delta(X)$ and $\sigma_{i}(X)$ as represent cost function, deflection and the behavioural constraints respectively whereas $\left[\sigma_{i}(X)\right]$ denotes the maximum allowable value , ' $m$ ' and ' $n$ ' are the number of constraints and design variables respectively. A given set of discrete value is expressed by $R^{d}$ and in this paper objective functions are taken as

$C(X)=\sum_{t=1}^{T} c_{t} \prod_{n=1}^{m} x_{n}^{t n}$ and $\delta(X)$

and constraint are chosen to be stress of structures as follows

$\sigma_{i}(X) \lesssim \sigma_{i}$ with allowable tolerance $\sigma_{i}^{0}$ for $i=1,2, \ldots, m$

Where $c_{t}$ is the cost coefficient of $\mathrm{t}^{\text {th }}$ side and $x_{n}$ is the $n^{\text {th }}$ design variable respectively, $m$ is the number of structural element, $\sigma_{i}$ and $\sigma_{i}^{0}$ are the $i^{\text {th }}$ stress, allowable stress respectively.

\section{MATHEMATICAL PRELIMINARIES}

\subsection{Fuzzy Set}

Let $X$ denotes a universal set. Then the fuzzy subset $A$ in $X$ is a subset of order pairs $\tilde{A}=\left\{\left(x, \mu_{\tilde{A}}(x)\right): x \in X\right\}$ where $\mu_{\tilde{A}}: X \rightarrow[0,1]$ is called the membership function which assigns a real number $\mu_{\tilde{A}}(x)$ in the interval $[0,1]$ to each element $x \in X . A$ is non fuzzy and $\mu_{\tilde{A}}(x)$ is identical to the characteristic function of crisp set.It is clear that the range of membership function is a subset of non-negative real numbers.

\section{2. $\alpha$ - Level set or $\alpha$ - cut of a fuzzy set}

The $\alpha$-level set of a fuzzy set $A$ of $X$ is a crisp set $A_{\alpha}$ which contains all the elements of $X$ that have membership values greater than or equal to $\alpha$ i.e $A=\left\{x: \mu_{A}(x) \geq \alpha, x \in X, \alpha \in[0,1]\right\}$.

\subsection{Intuitionistic Fuzzy Set}


Let $X=\left\{x_{1}, x_{2}, \ldots, x_{n}\right\}$ be a finite universal set. An intuitionistic fuzzy set (IFS) set $A^{i}$ in the sense of Attanassove [14] is given by equation $\tilde{A}^{i}=\left\{<X, \mu_{\tilde{A}^{i}}(x), v_{\tilde{A}^{i}}(x)>\mid x_{i} \in X\right\} \quad$ where $\quad$ the function $\quad \mu_{\tilde{A}^{i}}\left(x^{i}\right): X \rightarrow[0,1]$; $x_{i} \in X \rightarrow \mu_{\tilde{A}^{i}}\left(x_{i}\right) \in[0,1]$ and $\quad v_{\tilde{A}^{i}}\left(x^{i}\right): X \rightarrow[0,1] ; x_{i} \in X \rightarrow v_{A^{i}}\left(x_{i}\right) \in[0,1]$ define the degree of membership and degree of non-membership of an element $x_{i} \in X$ to the set $\tilde{A}^{i} \subseteq X$, such that they satisfy the condition $0 \leq \mu_{\tilde{A}^{i}}\left(x_{i}\right)+v_{\tilde{A}^{i}}\left(x_{i}\right) \leq 1, \forall x_{i} \in X$. For each IFS $\tilde{A}^{i}$ in $X$ the amount $\Pi_{\tilde{A}^{i}}\left(x_{i}\right)=1-\left(\mu_{\tilde{A}^{i}}\left(x^{i}\right)+v_{\tilde{A}^{i}}\left(x^{i}\right)\right)$ is called the degree of uncertainty (or hesitation) associated with the membership of elements $x_{i} \in X$ in $\tilde{A}^{i}$ we call it intuitionistic fuzzy index of $\tilde{A}^{i}$ with respect of an element $x_{i} \in X$.

\section{4. $(\alpha, \beta)$ cut of a Intuitionistic Fuzzy Set}

A set of $(\alpha, \beta)$ - cut ,generated by IFS $\tilde{A}^{i}$ where $\alpha, \beta \in[0,1]$ are fixed number such that $\alpha+\beta \leq 1$ is denoted by $\tilde{A}_{\alpha, \beta}^{i}=\left\{<x, \mu_{\tilde{A}^{i}}(x), v_{\tilde{A}^{i}}(x)>: x \in X, \mu_{\tilde{A}^{i}}(x) \geq \alpha, v_{\tilde{A}^{i}}(x) \leq \beta, \alpha, \beta \in[0,1]\right\}$ and defined as the crisp set of element $x$ which belong to $\tilde{A}^{i}$ at least to the degree $\alpha$ and which belong to $\tilde{A}^{i}$ at most to the degree $\beta$.

\subsection{Triangular Norm (T-Norm)}

$T:[0,1] \times[0,1] \rightarrow[0,1]$ is said to be t-norm if it satisfies the following properties

i) $T(a, b)=T(b, a) \forall a, b \in[0,1]$ (commutativity)

ii) $T(T(a, b), c)=T(a, T(b, c)) \forall a, b, c \in[0,1]$ (Associativity)

iii) $T(a, b) \leq T(a, c)$ with $b \leq c \quad \forall a, b, c \in[0,1]$ (Monotonocity)

iv) $T(0,0)=0, T(1,1)=1$;

v) $T(a, 1)=a \forall a \in[0,1]$

(Identity)

\subsection{Extended n-ary Triangular Norm (T-Norm)}

For the purpose of operations of multiple fuzzy sets ,it is useful to define the notation of multidimensional t-norms. Let $[0,1]^{n}$ be a $n$-dimensional cube and $\left(x_{1}, x_{2}, \ldots . ., x_{n}\right)$ 
and $\left(z_{1}, z_{2}, \ldots ., z_{n}\right) \in[0,1]^{n}$.A mapping $T:[0,1]^{n} \rightarrow[0,1]$ is called n-dimensional tnorm if it satisfies the following properties.

i) $T\left(x_{1}, x_{2}, \ldots . ., x_{i-1}, x_{i}, x_{i+1}, \ldots . ., x_{n}\right)=T\left(x_{1}, x_{2}, \ldots . ., x_{i-1}, x_{j}, x_{i+1}, \ldots ., x_{j-1}, x_{i}, x_{j+1}, \ldots ., x_{n}\right)$

ii) $T\left(T\left(x_{1}, x_{2}, \ldots . ., x_{n-1}, x_{n}\right), x_{n+1}, x_{n+2}, \ldots . ., x_{2 n-1}\right)=T\left(x_{1}, x_{2}, \ldots . ., x_{n-1}, T\left(x_{n}, x_{n+1}, \ldots ., x_{2 n-1}\right)\right)$

iii) For $\left(x_{1}, x_{2}, \ldots ., x_{n-1}, x_{n}\right) \leq\left(z_{1}, z_{2}, \ldots . ., z_{n-1}, z_{n}\right) \Rightarrow$

$T\left(x_{1}, x_{2}, \ldots ., x_{n-1}, x_{n}\right) \leq T\left(z_{1}, z_{2}, \ldots . ., z_{n-1}, z_{n}\right)$ with $x_{i}=z_{i}$ for some $i$ and $x_{i} \leq z_{i}$ for some $i=1,2, \ldots ., n$

iv) $T(0,0, \ldots \ldots \ldots ., 0)=0, T(1,1, \ldots \ldots \ldots ., 1)=1$

v) $T\left(1,1, \ldots . ., x_{i}, \ldots . ., 1\right)=x_{i}$

\subsection{Properties of Extended n-ary Triangular Norm (T-Norm)}

Due to associative law it is easy to extend a triangular norm $T$ into $\mathrm{n}$ arguments the n-ary operation $T_{n}$ on $[0,1]$ satisfies the following properties

i) $T_{n}\left(x_{1}, x_{2}, \ldots \ldots ., x_{n}\right)=T_{n}\left(x_{\sigma_{1}}, x_{\sigma_{2}}, \ldots \ldots ., x_{\sigma_{n}}\right)$ where $\sigma$ is a permutation of $\{1,2, \ldots \ldots, n\}$

(Commutativity)

ii) $T_{n}\left(x_{1}, x_{2}, \ldots \ldots, x_{n}\right)=T_{i+1}\left(x_{1}, x_{2}, \ldots ., x_{i}, T_{n-i}\left(x_{i+1}, \ldots, x_{j}, \ldots, x_{n}\right)\right)$

$=T_{n-j+1}\left(T\left(x_{1}, x_{2}, \ldots \ldots ., x_{j}\right), x_{j+1}, \ldots \ldots, x_{n}\right)$

iii) $\left(\forall i \in N_{n}\right)\left(x_{i} \leq x_{i}^{\prime}\right) \Rightarrow T_{n}\left(x_{1}, x_{2}, \ldots \ldots, x_{n}\right) \leq T_{n}\left(x_{1}^{\prime}, x_{2}^{\prime}, \ldots \ldots ., x_{n}^{\prime}\right)$

(monotonocity)

iv) $T_{n}\left(x_{1}, x_{2}, \ldots, x_{i-1}, 1, x_{i+1} \ldots ., x_{n}\right)=T\left(x_{1}, x_{2}, \ldots ., x_{i-1}, x_{i+1}, . ., x_{j}, \ldots, x_{n}\right)$

(Identity Law)

A t-norm $T_{n}$ is said to be continuous if $T$ is continuous function on[0,1].From the above lemma 1,we may call $T_{n}$ an extension of triangular norm .In the sequel we omit number of argument $\mathrm{n}$ and simply write $T$ of the class of mapping generated by triangular norm $T$.

\subsection{Triangular Conorm (T-Conorm)}

$S:[0,1] \times[0,1] \rightarrow[0,1]$ is said to be t-conorm if it satisfies the following properties

i) $S(a, b)=S(b, a) \forall a, b \in[0,1] \quad$ (commutativity) 

ii) $S(S(a, b), c)=S(a, S(b, c)) \forall a, b, c \in[0,1]$
(Associativity)
iii) $S(a, b) \leq S(a, c)$ with $b \leq c \quad \forall a, b, c \in[0,1]$
(Monotonocity)
iv) $S(0,0)=0, S(1,1)=1$;
v) $S(a, 0)=a \forall a \in[0,1]$
(Identity)

\subsection{Extended n-ary Triangular Conorm (T-Conorm)}

For the purpose of operations of multiple fuzzy sets ,it is useful to define the notation of multidimensional t-norms. Let $[0,1]^{n}$ be a n-dimensional cube and $\left(x_{1}, x_{2}, \ldots . ., x_{n}\right)$ and $\left(z_{1}, z_{2}, \ldots . ., z_{n}\right) \in[0,1]^{n}$.A mapping $S:[0,1]^{n} \rightarrow[0,1]$ is called n-dimensional $\mathrm{t}$ norm if it satisfies the following properties.

i) $S\left(x_{1}, x_{2}, \ldots . ., x_{i-1}, x_{i}, x_{i+1}, \ldots . ., x_{n}\right)=S\left(x_{1}, x_{2}, \ldots . ., x_{i-1}, x_{j}, x_{i+1}, \ldots ., x_{j-1}, x_{i}, x_{j+1}, \ldots ., x_{n}\right)$

ii) $S\left(S\left(x_{1}, x_{2}, \ldots . ., x_{n-1}, x_{n}\right), x_{n+1}, x_{n+2}, \ldots . ., x_{2 n-1}\right)=S\left(x_{1}, x_{2}, \ldots ., x_{n-1}, S\left(x_{n}, x_{n+1}, \ldots ., x_{2 n-1}\right)\right)$

iii) For $\left(x_{1}, x_{2}, \ldots . ., x_{n-1}, x_{n}\right) \leq\left(z_{1}, z_{2}, \ldots . ., z_{n-1}, z_{n}\right) \Rightarrow S\left(x_{1}, x_{2}, \ldots . ., x_{n-1}, x_{n}\right) \leq S\left(z_{1}, z_{2}, \ldots . ., z_{n-1}, z_{n}\right)$

with $x_{i}=z_{i}$ for some $i$ and $x_{i} \leq z_{i}$ for some $i=1,2, \ldots, n$

iv) $S(0,0, \ldots \ldots \ldots, 0)=0, S(1,1, \ldots \ldots \ldots, 1)=1$

v) $S\left(0,0, \ldots . ., x_{i}, \ldots . ., 0\right)=x_{i}$

\subsection{Properties of Extended n-ary Triangular Conorm (T-Norm)}

Due to associative law it is easy to extend a triangular norm $S$ into n arguments the n-ary operation $S_{n}$ on $[0,1]$ satisfies the following properties

i) $S_{n}\left(x_{1}, x_{2}, \ldots \ldots ., x_{n}\right)=S_{n}\left(x_{\sigma_{1}}, x_{\sigma_{2}}, \ldots \ldots, x_{\sigma_{n}}\right)$ where $\sigma$ is a permutation of $\{1,2, \ldots \ldots, n\}$

(Commutativity)

ii) $S_{n}\left(x_{1}, x_{2}, \ldots \ldots ., x_{n}\right)=S_{i+1}\left(x_{1}, x_{2}, \ldots ., x_{i}, S_{n-i}\left(x_{i+1}, . ., x_{j}, \ldots, x_{n}\right)\right)$

$=S_{n-j+1}\left(S\left(x_{1}, x_{2}, \ldots \ldots ., x_{j}\right), x_{j+1}, \ldots \ldots, x_{n}\right)$

iii) $\left(\forall i \in N_{n}\right)\left(x_{i} \leq x_{i}^{\prime}\right) \Rightarrow S_{n}\left(x_{1}, x_{2}, \ldots \ldots ., x_{n}\right) \leq S_{n}\left(x_{1}^{\prime}, x_{2}^{\prime}, \ldots \ldots, x_{n}^{\prime}\right) \quad$ (monotonocity)

iv) $S_{n}\left(x_{1}, x_{2}, \ldots, x_{i-1}, 0, x_{i+1} \ldots, x_{n}\right)=S\left(x_{1}, x_{2}, \ldots, x_{i-1}, x_{i+1}, . ., x_{j}, \ldots, x_{n}\right) \quad$ (Identity Law)

A t-norm $S_{n}$ is said to be continuous if $S$ is continuous function on [0,1].From the above lemma 1,we may call $S_{n}$ an extension of triangular norm .In the sequel we omit 
number of argument $\mathrm{n}$ and simply write $S$ of the class of mapping generated by triangular norm $S$.

\subsection{Four Basic T-norm and T-conorm and their Generalization with Weight} Factors

Let $\tilde{A}_{j}^{i}=\left\{<x_{j}, \mu_{\tilde{A}_{j}^{i}}\left(x_{j}\right), v_{\tilde{A}_{j}^{i}}\left(x_{j}\right)>: x_{j} \in X, \mu_{\tilde{A}_{j}^{i}}\left(x_{j}\right) \geq \alpha, v_{\tilde{A}_{j}^{i}}\left(x_{j}\right) \leq \beta, \alpha, \beta \in[0,1]\right\}$ be $\mathrm{n}$ intuitionistic fuzzy set for $j=1,2, \ldots ., n$.

\section{i) Minimum t-norm and maximum t-conorm}

The intuitionistic fuzzy minimum t-norm and maximum t-co-norm can be defined as $T_{M}\left(\mu_{1}\left(x_{1}\right), \mu_{2}\left(x_{2}\right), \ldots . ., \mu_{n}\left(x_{n}\right)\right)=\min \left\{\mu_{1}\left(x_{1}\right), \mu_{2}\left(x_{2}\right), \ldots . ., \mu_{n}\left(x_{n}\right)\right\}$ and $S_{M}\left(v_{1}\left(x_{1}\right), v_{2}\left(x_{2}\right), \ldots . ., v_{n}\left(x_{n}\right)\right)=\max \left\{v_{1}\left(x_{1}\right), v_{2}\left(x_{2}\right), \ldots . ., v_{n}\left(x_{n}\right)\right\}$

Similarly n-ary intuitionistic fuzzy minimum t-norm and maximum t-co-norm with weight can be defined as $T_{M}^{w}\left(w_{1}, \mu_{1}\left(x_{1}\right) ; w_{2}, \mu_{2}\left(x_{2}\right) ; \ldots . . ; w_{n}, \mu_{n}\left(x_{n}\right)\right)=\min \left\{w_{1} \mu_{1}\left(x_{1}\right) ; w_{2} \mu_{2}\left(x_{2}\right) ; \ldots . . ; w_{n} \mu_{n}\left(x_{n}\right)\right\}$ and $S_{M}^{w}\left(w_{1}, v_{1}\left(x_{1}\right) ; w_{2}, v_{2}\left(x_{2}\right) ; \ldots . ; w_{n}, v_{n}\left(x_{n}\right)\right)=\max \left\{w_{1} v_{1}\left(x_{1}\right) ; w_{2} v_{2}\left(x_{2}\right) ; \ldots . . ; w_{n} v_{n}\left(x_{n}\right)\right\}$

\section{ii) Probabilistic t-norm and t-conorm}

The intuitionistic fuzzy probabilistic t-norm and t-co-norm can be defined as $T_{P}\left(\mu_{1}\left(x_{1}\right), \mu_{2}\left(x_{2}\right), \ldots . ., \mu_{n}\left(x_{n}\right)\right)=\prod_{i=1}^{n}\left(\mu_{i}\left(x_{i}\right)\right)$ and
$S_{P}\left(v_{1}\left(x_{1}\right), v_{2}\left(x_{2}\right), \ldots . ., v_{n}\left(x_{n}\right)\right)=1-\prod_{i=1}^{n}\left(1-v_{i}\left(x_{i}\right)\right)$

Similarly n-ary intuitionistic fuzzy probabilistic t-norm and t-co-norm with weight can be defined as

$$
\begin{aligned}
& T_{P}^{w}\left(w_{1}, \mu_{1}\left(x_{1}\right) ; w_{2}, \mu_{2}\left(x_{2}\right) ; \ldots . ; w_{n}, \mu_{n}\left(x_{n}\right)\right)=\prod_{i=1}^{n}\left(\mu_{i}\left(x_{i}\right)\right)^{w_{i}} \text { and } \\
& S_{P}^{w}\left(w_{1}, v_{1}\left(x_{1}\right) ; w_{2}, v_{2}\left(x_{2}\right) ; \ldots . . ; w_{n}, v_{n}\left(x_{n}\right)\right)=1-\prod_{i=1}^{n}\left(1-v_{i}\left(x_{i}\right)\right)^{w_{i}}
\end{aligned}
$$

iii) Lukasewicz t-norm and t-conorm

The intuitionistic fuzzy Lukasewicz t-norm and t-co-norm can be defined as 


$$
\begin{aligned}
& T_{L}\left(\mu_{1}\left(x_{1}\right), \mu_{2}\left(x_{2}\right), \ldots . ., \mu_{n}\left(x_{n}\right)\right)=\max \left(\sum_{i=1}^{n} \mu_{i}\left(x_{i}\right)-(n-1), 0\right) \text { and } \\
& S_{L}\left(v_{1}\left(x_{1}\right), v_{2}\left(x_{2}\right), \ldots . ., v_{n}\left(x_{n}\right)\right)=\min \left\{1, \sum_{i=1}^{n} v_{i}\left(x_{i}\right)\right\}
\end{aligned}
$$

Similarly n-ary intuitionistic fuzzy Lukasewicz t-norm and t-co-norm with weight can be defined as

$$
\begin{aligned}
& T_{L}^{w}\left(\mu_{1}\left(x_{1}\right), \mu_{2}\left(x_{2}\right), \ldots \ldots, \mu_{n}\left(x_{n}\right)\right)=\max \left(\sum_{i=1}^{n} w_{i} \mu_{i}\left(x_{i}\right)-(n-1), 0\right) \text { and } \\
& S_{L}^{w}\left(v_{1}\left(x_{1}\right), v_{2}\left(x_{2}\right), \ldots . ., v_{n}\left(x_{n}\right)\right)=\min \left\{1, \sum_{i=1}^{n} w_{i} v_{i}\left(x_{i}\right)\right\}
\end{aligned}
$$

iv)Weber (or Drastic Product) $t$-norm and t-conorm

The intuitionistic fuzzy Weber (or Drastic Product ) t-norm and t-co-norm can be defined as

$T_{D}\left(\mu_{1}\left(x_{1}\right), \mu_{2}\left(x_{2}\right), \ldots . ., \mu_{n}\left(x_{n}\right)\right)=\left\{\begin{array}{cl}\min \left(\mu_{i}\left(x_{i}\right)\right) & \text { if } \max \left(\mu_{i}\left(x_{i}\right)\right)=1 \\ 0 & \text { otherwise }\end{array}\right.$

and $S_{D}\left(v_{1}\left(x_{1}\right), v_{2}\left(x_{2}\right), \ldots . ., v_{n}\left(x_{n}\right)\right)= \begin{cases}\max \left(v_{i}\left(x_{i}\right)\right) & \text { if } \min \left(v_{i}\left(x_{i}\right)\right)=0 \\ 1 & \text { if } \min \left(v_{i}\left(x_{i}\right)\right)>0\end{cases}$

Similarly n-ary intuitionistic fuzzy Lukasewicz t-norm and t-co-norm with weight can be defined as

$T_{D}^{w}\left(w_{1}, \mu_{1}\left(x_{1}\right) ; w_{2}, \mu_{2}\left(x_{2}\right) ; \ldots . ; w_{n}, \mu_{n}\left(x_{n}\right)\right)=\left\{\begin{array}{cl}\min \left(w_{i} \mu_{i}\left(x_{i}\right)\right) & \text { if } \max \left(w_{i} \mu_{i}\left(x_{i}\right)\right)=1 \\ 0 & \text { otherwise }\end{array}\right.$

and

$$
S_{D}^{w}\left(w_{1}, v_{1}\left(x_{1}\right) ; w_{2}, v_{2}\left(x_{2}\right) ; \ldots ., w_{n}, v_{n}\left(x_{n}\right)\right)=\left\{\begin{array}{cc}
\max \left(w_{i} v_{i}\left(x_{i}\right)\right) & \text { if } \min \left(w_{i} v_{i}\left(x_{i}\right)\right)=0 \\
1 & \text { if } \min \left(w_{i} v_{i}\left(x_{i}\right)\right)>0
\end{array}\right.
$$

\subsection{Some Particular Classes of T-norms and T-Co-norms}

A t-norm and t-co-norm is commutative order semi-group with unit element 1 on $[0,1]$ of real numbers, So the class of t-norm and t-co-norm is quite large. Two well-known class of t-norm and t-co-norm are discussed here.

Yager (1980) introduced the following classes of t-norms and t-co-norms as $T_{\lambda}^{Y}\left(\mu_{1}\left(x_{1}\right), \mu_{2}\left(x_{2}\right)\right)=1-\min \left[1,\left\{\left(1-\mu_{1}\left(x_{1}\right)\right)^{\lambda}+\left(1-\mu_{2}\left(x_{2}\right)\right)^{\lambda}\right\}^{\left(\frac{1}{\lambda}\right)}\right] \forall \lambda \in[0, \infty)$ 
and $S_{\lambda}^{Y}\left(v_{1}\left(x_{1}\right), v_{2}\left(x_{2}\right)\right)=\min \left[1,\left\{\left(v_{1}\left(x_{1}\right)\right)^{\lambda}+\left(v_{2}\left(x_{2}\right)\right)^{\lambda}\right\}^{\left(\frac{1}{\lambda}\right)}\right] \forall \lambda \in[0, \infty)$

Extended n-ary form of above t-norm is

$$
\begin{aligned}
& T_{\lambda}^{Y}\left(\mu_{1}\left(x_{1}\right), \mu_{1}\left(x_{2}\right), \ldots \ldots . ., \mu_{n}\left(x_{n}\right)\right)=1-\min \left[1,\left\{\sum_{i=1}^{n}\left(1-\mu_{i}\left(x_{i}\right)\right)^{\lambda}\right\}^{\frac{1}{\lambda}}\right] \forall \lambda \in[0, \infty) \\
& \text { and } S_{\lambda}^{Y}\left(v_{1}\left(x_{1}\right), v_{1}\left(x_{2}\right), \ldots \ldots . ., v_{n}\left(x_{n}\right)\right)=\min \left[1,\left\{\sum_{i=1}^{n}\left(v_{i}\left(x_{i}\right)\right)^{\lambda}\right\}^{\frac{1}{\lambda}}\right] \forall \lambda \in[0, \infty)
\end{aligned}
$$

The extended form with different weights of the above t-norms and t-conorms are $T_{\lambda}^{Y}\left(w_{1}, \mu_{1}\left(x_{1}\right) ; w_{2}, \mu_{1}\left(x_{2}\right) ; \ldots \ldots . ., w_{n}, \mu_{n}\left(x_{n}\right)\right)=1-\min \left[1,\left\{\sum_{i=1}^{n}\left(1-w_{i} \mu_{i}\left(x_{i}\right)\right)^{\lambda}\right\}^{\frac{1}{\lambda}}\right] \forall \lambda \in[0, \infty)$ and

$$
S_{\lambda}^{Y}\left(w_{1}, v_{1}\left(x_{1}\right) ; w_{2}, v_{1}\left(x_{2}\right) ; \ldots \ldots . . ; w_{n}, v_{n}\left(x_{n}\right)\right)=\min \left[1,\left\{\sum_{i=1}^{n} w_{i}\left(v_{i}\left(x_{i}\right)\right)^{\lambda}\right\}^{\frac{1}{\lambda}}\right] \forall \lambda \in[0, \infty)
$$

Hamacher (1978) introduced the following classes of t-norms and t-co-norms as $T_{\lambda}^{H}\left(\mu_{1}\left(x_{1}\right), \mu_{2}\left(x_{2}\right)\right)=\frac{\mu_{1}\left(x_{1}\right) \mu_{2}\left(x_{2}\right)}{\lambda+(1-\lambda)\left\{1-\left(1-\mu_{1}\left(x_{1}\right)\right)\left(1-\mu_{2}\left(x_{2}\right)\right)\right\}} \quad \forall \lambda \in[0, \infty)$ and $S_{\lambda}^{H}\left(v_{1}\left(x_{1}\right), v_{2}\left(x_{2}\right)\right)=\frac{v_{1}\left(x_{1}\right)+v_{2}\left(x_{2}\right)-(2-\lambda) v_{1}\left(x_{1}\right) v_{2}\left(x_{2}\right)}{1-(1-\lambda) v_{1}\left(x_{1}\right) v_{2}\left(x_{2}\right)} \quad \lambda \geq 0$

Extended n-ary form of above t-norm is

$$
\begin{aligned}
& T_{\lambda}^{H}\left(\mu_{1}\left(x_{1}\right), \mu_{2}\left(x_{2}\right), \ldots \ldots, \mu_{n}\left(x_{n}\right)\right)=\frac{\prod_{i=1}^{n} \mu_{i}\left(x_{i}\right)}{\lambda+(1-\lambda)\left\{1-\prod_{i=1}^{n}\left(1-\mu_{i}\left(x_{i}\right)\right)\right\}} \forall \lambda \in[0, \infty) \\
& \text { and } S_{\lambda}^{H}\left(v_{1}\left(x_{1}\right), v_{1}\left(x_{2}\right), \ldots \ldots ., v_{n}\left(x_{n}\right)\right)=\frac{\sum_{i=1}^{n} v_{i}\left(x_{i}\right)-(2-\lambda) \prod_{i=1}^{n} v_{i}\left(x_{i}\right)}{1-(1-\lambda) \prod_{i=1}^{n} v_{i}\left(x_{i}\right)} \quad \lambda \geq 0
\end{aligned}
$$


The extended form with different weights of the above t-norms and t-conorms are

$$
T_{\lambda}^{H}\left(w_{1}, \mu_{1}\left(x_{1}\right) ; w_{2}, \mu_{2}\left(x_{2}\right) ; \ldots \ldots ; w_{n}, \mu_{n}\left(x_{n}\right)\right)=\frac{\prod_{i=1}^{n}\left(\mu_{i}\left(x_{i}\right)\right)^{w_{i}}}{\lambda+(1-\lambda)\left\{1-\prod_{i=1}^{n}\left(1-\mu_{i}\left(x_{i}\right)\right)^{w_{i}}\right\}} \forall \lambda \in[0, \infty)
$$

and

$$
S_{\lambda}^{H}\left(w_{1}, v_{1}\left(x_{1}\right) ; w_{1}, v_{1}\left(x_{2}\right) ; \ldots \ldots . . ; w_{n}, v_{n}\left(x_{n}\right)\right)=\frac{\sum_{i=1}^{n} w_{i} v_{i}\left(x_{i}\right)-(2-\lambda) \prod_{i=1}^{n}\left(v_{i}\left(x_{i}\right)\right)^{w_{i}}}{1-(1-\lambda) \prod_{i=1}^{n}\left(v_{i}\left(x_{i}\right)\right)^{w_{i}}} \lambda \geq 0
$$

\section{WEIGHTED INTUITIONISTIC FUZZY AGGREGATION}

Weighted aggregation has been used quiet extensively especially in fuzzy decision making, where the weight are used to represent the relative importance and the negligence the decision maker attaches to different decision criterion (goals or constraints). Weighted aggregation of fuzzy sets by using t-norm has been considered by Yagar (1978) .He proposed to modify the membership function with the associated weight factors before the fuzzy aggregation.Xeshui Xu (2007) presented intuitionistic fuzzy aggregation operator. The weighted aggregation is then the aggregation of the modified membership and non-membership functions and the general form of this idea is

$$
\begin{aligned}
& D_{1}(x, w)=T\left(I\left(\mu_{1}\left(x_{1}\right), w_{1}\right), I\left(\mu_{2}\left(x_{2}\right), w_{2}\right), \ldots \ldots \ldots, I\left(\mu_{k}\left(x_{k}\right), w_{k}\right)\right) \\
& D_{2}(x, w)=S\left(I\left(v_{1}\left(x_{1}\right), w_{1}\right), I\left(v_{2}\left(x_{2}\right), w_{2}\right), \ldots \ldots \ldots, I\left(v_{k}\left(x_{k}\right), w_{k}\right)\right)
\end{aligned}
$$

Where $w$ are vectors of weight factor $w_{i} \in[0,1] i=1,2, \ldots ., k$ associated with the aggregated membership function $\mu_{i}\left(x_{i}\right)$ and non-membership function $v_{i}\left(x_{i}\right)$. Here $\mathrm{T}$ is triangular norm and $\mathrm{S}$ is triangular conorm , $\mathrm{I}$ is a function of two variables that transforms the membership and non-membership with $\sum_{i=1}^{k} w_{i}=1, w_{i} \geq 0$;

\section{INTUITIONISTIC FUZZY NON-LINEAR PROGRAMMING (IFNLP) OPTIMIZATION WITH DIFFERENT WEIGHTED T-NORM AND T- CONORM OPERATOR TO SOLVE MULTI-OBJECTIVE NON-LINEAR PROGRAMMING PROBLEM (PMONLP)}

A multi-objective non-linear parametric intuitionistic programming (MONLP)Problem can be formulated as 


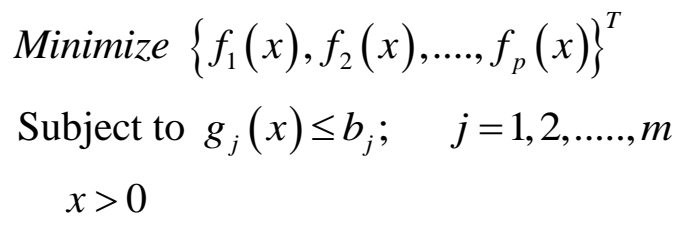

Following Zimmermann (1978),we have presented a solution algorithm to solve the MONLP Problem by fuzzy optimization technique.

Step-1: Solve the MONLP (2) as a single objective non-linear programming problem $p$ tiby taking one of the objective at a time and ignoring the others. These solutions areknown as ideal solutions. Let $x^{i}$ be the respective optimal solution for the $i^{\text {th }}$ different objectives with same constraints and evaluate each objective values for all these $i^{\text {th }}$ optimal solutions.

Step-2: From the result of step -1 determine the corresponding values for every objective for each derived solutions. With the values of all objectives at each ideal solutions , pay-off matrix can be formulated as follows

$$
\begin{aligned}
& f_{1}(x) \quad f_{2}(x) \quad \ldots \ldots . . . \quad f_{p}(x) \\
& \begin{array}{c}
x^{1} \\
x^{2} \\
\vdots \\
x^{p}
\end{array}\left[\begin{array}{cccc}
f_{1}^{*}\left(x^{1}\right) & f_{2}^{*}\left(x^{1}\right) & \ldots \ldots \ldots & f_{p}^{*}\left(x^{1}\right) \\
f_{1}^{*}\left(x^{2}\right) & f_{2}^{*}\left(x^{2}\right) & \ldots \ldots \ldots & f_{p}^{*}\left(x^{2}\right) \\
\ldots \ldots \ldots \ldots & \ldots \ldots \ldots \ldots \ldots & \ldots \ldots \ldots & \ldots \ldots \ldots \ldots \\
f_{1}^{*}\left(x^{p}\right) & f_{2}^{*}\left(x^{p}\right) & \ldots \ldots \ldots & f_{p}^{*}\left(x^{p}\right)
\end{array}\right]
\end{aligned}
$$

Here $x^{1}, x^{2}, \ldots \ldots, x^{p}$ are the ideal solution of the objectives $f_{1}(x), f_{2}(x), \ldots ., f_{p}(x)$ respectively.

Step-3: From the result of step 2 now we find lower bound (minimum) $L_{i}^{A C C}$ and upper bound (maximum) $U_{i}^{A C C}$ by using following rule $U_{i}^{A C C}=\max \left\{f_{i}\left(x^{p}\right)\right\}, L_{i}^{A C C}=\min \left\{f_{i}\left(x^{p}\right)\right\}$ where $1 \leq i \leq p$. But in IFO The degree of non-membership (rejection) and the degree of membership (acceptance) are considered so that the sum of both value is less than one. To define the non membership of NLP problem let $U_{i}^{\mathrm{Re} j}$ and $L_{i}^{\operatorname{Re} j}$ be the upper bound and lower bound of objective function $f_{i}(x)$ where $L_{i}^{A C C} \leq L_{i}^{\operatorname{Re} j} \leq U_{i}^{\operatorname{Re} j} \leq U_{i}^{A C C}$. For objective function of minimization problem , the upper bound for non-membership function (rejection) is always equals to that the upper bound of membership function (acceptance). One can take lower bound for non-membership function as follows $L_{i}^{\mathrm{Re} j}=L_{i}^{A c c}+\varepsilon_{i}$ where $0<\varepsilon_{i}<\left(U_{i}^{A c c}-L_{i}^{A c c}\right)$ based on the decision maker choice. 
The initial intuitionistic fuzzy model with aspiration level of objectives becomes Find

$$
\left\{x_{i}, i=1,2, \ldots, p\right\}
$$

so as to satisfy $f_{i}(x) \leq L_{i}^{A c c}$ with tolerance $P_{i}^{A c c}=\left(U_{i}^{A c c}-L_{i}^{A c c}\right)$ for the degree of acceptance for $i=1,2, \ldots . ., p . \quad f_{i}(x) \geq^{i} U_{i}^{\operatorname{Re} j}$ with tolerance $P_{i}^{A c c}=\left(U_{i}^{A c c}-L_{i}^{A c c}\right)$ for degree of rejection for $i=1,2, \ldots . ., p$.Define the membership (acceptance) and nonmembership (rejection) functions of above uncertain objectives as follows. For the $i^{t h}, i=1,2, \ldots, p$ objectives functions the linear membership function $\mu_{i}\left(f_{i}(x)\right)$ and linear non-membership $v_{i}\left(f_{i}(x)\right)$ is defined as follows

$$
\begin{aligned}
& \mu_{i}\left(f_{i}(x)\right)=\left\{\begin{array}{ccc}
1 & \text { if } & f_{i}(x) \leq L_{i}^{A c c} \\
\frac{e^{-T\left(\frac{f_{i}(x)-L_{i}^{A c c}}{U_{i}^{A c c}-L_{i}^{A c c}}\right)}-e^{-T}}{1-e^{-T}} & \text { if } & L_{i}^{A c c} \leq f_{i}(x) \leq U_{i}^{A c c} \\
0 & \text { if } & f_{i}(x) \geq U_{i}^{A c c}
\end{array}\right. \\
& v_{i}\left(f_{i}(x)\right)=\left\{\begin{array}{ccc}
0 & \text { if } & f_{i}(x) \leq L_{i}^{\operatorname{Re} j} \\
\left(\frac{f_{i}(x)-L_{i}^{\operatorname{Re} j}}{\left.U_{i}^{\operatorname{Re} j}-L_{i}^{\operatorname{Re} j}\right)^{2}}\right. & \text { if } & L_{i}^{\operatorname{Re} j} \leq f_{i}(x) \leq U_{i}^{\operatorname{Re} j} \\
1 & \text { if } & f_{i}(x) \geq U_{i}^{\operatorname{Re} j}
\end{array}\right.
\end{aligned}
$$

After determining the different membership functions for each of the objective functions, one can adopt following three type of decisions

i)Intuitionistic Min-Max Operator, ii) Probabilistic t-norm and t-conorm Operator,iii) Lukasewicz t-norm and t-conorm Operator

i)According to the extension of the weighted intuitionistic min-max operator the MONLP (2)

can be formulated as

$$
\begin{aligned}
& \text { Maximize } \mu_{\tilde{D}_{1}}(x ; w)=\text { Maximize }\left(\text { Minimum }\left\{w_{1} \mu_{1}\left(f_{1}(x)\right), w_{2} \mu_{2}\left(f_{2}(x)\right), \ldots \ldots, w_{p} \mu_{p}\left(f_{p}(x)\right)\right\}\right) \\
& \text { Minimize } v_{\tilde{D}_{2}}(x ; w)=\operatorname{Minimize}\left(\operatorname{Maximum}\left\{w_{1} v_{1}\left(f_{1}(x)\right), w_{2} v_{2}\left(f_{2}(x)\right), \ldots \ldots, w_{p} v_{p}\left(f_{p}(x)\right)\right\}\right) \\
& \text { such that } \\
& 0 \leq \mu_{i}\left(f_{i}(x)\right)+v_{i}\left(f_{i}(x)\right) \leq 1 ; \text { for } i=1,2, \ldots, p . \\
& \mu_{i}\left(f_{i}(x)\right) \geq v_{i}\left(f_{i}(x)\right), \text { for } i=1,2, \ldots, p .
\end{aligned}
$$


$\mu_{i}\left(f_{i}(x)\right) \in[0,1], v_{i}\left(f_{i}(x)\right) \in[0,1]$, for $i=1,2, \ldots, p$.

$g_{j}(x) \leq b_{j} ; j=1,2, \ldots ., m$.

$x>0 ; \sum_{i=1}^{p} w_{i}=1 ; w_{i i} \in[0,1]$ for $i=1,2, \ldots, p$.

According to Angelov (1986) the above problem can be formulated as

Maximize $(\alpha-\beta)$

$w_{i} \mu_{i}\left(f_{i}(x)\right) \geq \alpha ; w_{i} v_{i}\left(f_{i}(x)\right) \leq \beta ;$ for $i=1,2, . ., p$.

$g_{j}(x) \leq b_{j} ;$ for $j=1,2, \ldots, m$.

$x>0 ; 0 \leq \alpha+\beta \leq 1 ; \alpha \geq \beta ; \alpha, \beta \in[0,1]$

$x>0 ; \sum_{i=1}^{p} w_{i}=1 ; w_{i} \in[0,1]$

ii)According to the extension of the weighted intuitionistic Probabilistic operator the MONLP (2) can be formulated as

Maximize $\mu_{\tilde{D}_{1}}(x ; w)=$ Maximize $\prod_{i=1}^{n}\left(\mu_{i}\left(x_{i}\right)\right)^{w_{i}}$

Minimize $v_{\tilde{D}_{2}}(x ; w)=\operatorname{Minimize}\left\{1-\prod_{i=1}^{n}\left(1-v_{i}\left(x_{i}\right)\right)^{w_{i}}\right\}$

Subject to the same constraint as (i)

iii)According to the extension of the weighted intuitionistic Lukasewicz operator the MONLP (2) can be formulated as

Maximize $\mu_{\tilde{D}_{1}}(x ; w)=\operatorname{Maximize}\left(\sum_{i=1}^{n} \mu_{i}\left(x_{i}\right)-(n-1), 0\right)$

Minimize $v_{\tilde{D}_{2}}(x ; w)=$ Minimize $\left\{1, \sum_{i=1}^{n} w_{i} v_{i}\left(x_{i}\right)\right\}$

Subject to the same constraint as (i)

iv)According to the extension of the weighted intuitionistic Yager (1980) operator the MONLP (2) can be formulated as

Maximize $\mu_{\tilde{D}_{1}}(x ; w)=$ Maximize $\left\{0,1-\sqrt[\lambda]{\sum_{i=1}^{p} w_{i}\left(1-\mu_{i}\left(f_{i}(x)\right)\right)^{\lambda}}\right\} \lambda>0$

Minimize $v_{\tilde{D}_{2}}(x ; w)=$ Minimize $\left\{1, \sqrt[\lambda]{\sum_{i=1}^{p} w_{i} v_{i}\left(f_{i}(x)\right)^{\lambda}}\right\} \lambda>0$, 
Subject to the same constraint as (i)

v)According to the extension of the weighted intuitionistic Hamacher (1978) operator the MONLP (2) can be formulated as

$$
\begin{gathered}
\text { Maximize } \mu_{\tilde{D}_{1}}(x ; w)=\text { Maximize } \frac{\prod_{i=1}^{p}\left(\mu_{i}\left(f_{i}(x)\right)\right)^{w_{i}}}{\lambda+(1-\lambda)\left\{1-\prod_{i=1}^{p}\left(1-\mu_{i}\left(f_{i}(x)\right)\right)^{w_{i}}\right\}} \lambda \leq 2 \\
\text { Minimize } v_{\tilde{D}_{2}}(x ; w)=\text { Minimize } \frac{\sum_{i=1}^{p} w_{i}\left(v_{i}\left(f_{i}(x)\right)\right)-(2-\lambda) \prod_{i=1}^{p}\left(v_{i}\left(f_{i}(x)\right)\right)^{w_{i}}}{1-(1-\lambda) \prod_{i=1}^{p}\left(v_{i}\left(f_{i}(x)\right)\right)^{w_{i}}} \lambda \geq 0,
\end{gathered}
$$

Subject to the same constraint as (i)

Step-4: Solving any of the above two we will get the optimal solution of MONLP (2).

\section{SOLUTION OF MULTI-OBJECTIVE WELDED BEAM OPTIMIZATION PROBLEM(MOWBOP) BY INTUITIONISTIC FUZZY OPTIMIZATION TECHNIQUE}

To solve the MOWBOP (1) step 1 of 5is used. After that according to step 2 pay-off matrix is formulated

$$
\begin{aligned}
& C(X) \quad \delta(X)
\end{aligned}
$$

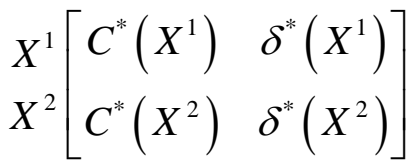

In next step following step 2 we calculate the bound of the objective $U_{C(X)}^{A c c}, L_{C(X)}^{A c c}$ and $U_{C(X)}^{\mathrm{Re} j}, L_{C(X)}^{\mathrm{Re} j}$ for weight function $C(X)$, such that $L_{C(X)}^{A c c}<C(X)<U_{C(X)}^{A c c}$ and $L_{C(X)}^{\mathrm{Re} j}<C(X)<U_{C(X)}^{\operatorname{Re} j}$ and $U_{\delta(X)}^{A c c}, L_{\delta(X)}^{A c c} ; U_{\delta(X)}^{\operatorname{Re} j}, L_{\delta(X)}^{\operatorname{Re} j}$ for deflection $\delta(X)$, such that $L_{\delta(X)}^{A c c}<\delta(X)<U_{\delta(X)}^{A c c}$ and $L_{\delta(X)}^{\operatorname{Re} j}<\delta(X)<U_{\delta(X)}^{\operatorname{Re} j}$ with the condition $\quad U_{i}^{A c c}=U_{i}^{\operatorname{Re} j} ;$ $L_{i}^{\mathrm{Re} j}=L_{i}^{A c c}+\varepsilon_{i} \quad$ for $i=\delta(X), C(X)$ so as $\quad 0<\varepsilon_{i}<\left(U_{i}^{A c c}-L_{i}^{A c c}\right)$ are identified.

According to IFO technique considering membership and non-membership function for (MOWBOP) 


$$
\begin{aligned}
& \mu_{C(X)}(C(X))=\left\{\begin{array}{ccc}
1 & \text { if } & C(X) \leq L_{C(X)}^{A c c} \\
\frac{e^{-T\left(\frac{C(X)-L_{C(X)}^{A c c}}{U_{C(X)}^{A c-}-L_{C(X)}^{A c c}}\right)}-e^{-T}}{1-e^{-T}} & \text { if } & L_{C(X)}^{A c c} \leq C(X) \leq U_{C(X)}^{A c c} \\
0 & \text { if } \quad C(X) \geq U_{C(X)}^{A c c}
\end{array}\right. \\
& v_{C(X)}(C(X))=\left\{\begin{array}{ccc}
0 & \text { if } & C(X) \leq L_{C(X)}^{\mathrm{Re} j} \\
\left(\frac{C(X)-L_{C(X)}^{\mathrm{Re} j}}{U_{C(X)}^{\operatorname{Re} j}-L_{C(X)}^{\operatorname{Re} j}}\right)^{2} & \text { if } & L_{C(X)}^{\mathrm{Re} j} \leq C(X) \leq U_{C(X)}^{\mathrm{Re} j} \\
1 & \text { if } & C(X) \geq U_{C(X)}^{\operatorname{Re} j}
\end{array}\right.
\end{aligned}
$$

And

$$
\begin{aligned}
& \mu_{\delta(X)}(\delta(X))=\left\{\begin{array}{ccc}
1 & \text { if } \delta(X) \leq L_{\delta(X)}^{A c c} \\
\frac{e^{-T\left(\frac{\delta(X)-L_{\delta(X)}^{A c c}}{U_{\delta(X)}^{A c c}-L_{\delta(X)}^{A c c}}\right)}-e^{-T}}{1-e^{-T}} & \text { if } & L_{\delta(X)}^{A c c} \leq \delta(X) \leq U_{\delta(X)}^{A c c} \\
0 & \text { if } \quad & \delta(X) \geq U_{\delta(X)}^{A c c}
\end{array}\right. \\
& v_{\delta(X)}(\delta(X))=\left\{\begin{array}{ccc}
0 & \text { if } & \delta(X) \leq L_{\delta(X)}^{\mathrm{Re} j} \\
\left(\frac{\delta(X)-L_{\delta(X)}^{\mathrm{Re} j}}{U_{\delta(X)}^{\operatorname{Re} j}-L_{\delta(X)}^{\mathrm{Re} j}}\right)^{2} & \text { if } & L_{\delta(X)}^{\mathrm{Re} j} \leq \delta(X) \leq U_{\delta(X)}^{\mathrm{Re} j} \\
1 & \text { if } & \delta(X) \geq U_{\delta(X)}^{\mathrm{Re} j}
\end{array}\right.
\end{aligned}
$$

After determining the different membership functions for each of the objective functions, one can adopt following five type of decisions

6 i) According to Min-Max operator the MOWBOP can be formulated as

Maximize $(\alpha-\beta)$

$w_{1} \mu_{C(X)}(C(X)) \geq \alpha$;

$w_{1} v_{C(X)}(C(X)) \leq \beta$

$w_{2} \mu_{\delta(X)}(\delta(X)) \geq \alpha$; 
$w_{2} \mu_{\delta(X)}(\delta(X)) \leq \beta ;$

$\sigma_{i}(X) \leq\left[\sigma_{i}\right]$;

$X^{\min } \leq X \leq X^{\max }$

$w_{1} \geq 0, w_{2} \geq 0, w_{1}+w_{2}=1 ; w_{1}, w_{2} \in[0,1] \alpha+\beta \leq 1 ; \alpha, \beta \in[0,1]$

6.ii)According to extension of weighted Probabilistic operator

Maximize $\left\{\mu_{C}(C(X))^{w_{1}} \mu_{\delta(X)}(\delta(X))^{w_{2}}\right\}$

Minimize $\left[1-\left(\left\{1-v_{C(X)}(C(X))\right\}^{w_{1}}\left\{1-v_{\delta(X)}(\delta(X))\right\}^{w_{2}}\right)\right]$

such that

$0 \leq \mu_{C(X)}(C(X))+v_{C(X)}(C(X)) \leq 1$;

$0 \leq \mu_{\delta(X)}(\delta(X))+v_{\delta(X)}(\delta(X)) \leq 1$;

$\mu_{C(X)}(C(X)) \geq v_{C(X)}(C(X))$;

$\mu_{\delta(X)}(\delta(X)) \geq v_{\delta(X)}(\delta(X))$;

$\sigma_{i}(X) \leq\left[\sigma_{i}\right]$

$X^{\min } \leq X \leq X^{\max }$

$w_{1} \geq 0, w_{2} \geq 0, w_{1}+w_{2}=1 ; w_{1}, w_{2} \in[0,1]$

6.iii)According to extension of weighted Lukasewicz operator

Maximize $\left\{w_{1} \mu_{C(X)}(C(X))+w_{2} \mu_{\delta(X)}(\delta(X))-1\right\}$

Minimize $\left\{w_{1} v_{C(X)}(C(X))+w_{2} v_{\delta(X)}(\delta(X))\right\}$

subject to the same constraint as (6.ii)

6.iv)According to extension of weighted Yagar operator with $\lambda=2$

Maximize $\left\{0,1-\sqrt{w_{1}\left(1-\mu_{C(X)}(C(X))\right)^{2}+w_{2}\left(1-\mu_{\delta(X)}(\delta(X))\right)^{2}}\right\}$

Minimize $\left\{1, \sqrt{w_{1} v_{C(X)}(C(X))^{2}+w_{2} v_{\delta(X)}(\delta(X))^{2}}\right\}$

subject to the same constraint as (6.ii)

6.v)According to extension of weighted Hamacher operator with $\lambda=1.5$ 
Maximize $\frac{\left\{\mu_{C(X)}(C(X))\right\}^{w_{1}}\left\{\mu_{\delta(X)}(\delta(X))\right\}^{w_{2}}}{1.5-0.5\left[\left\{1-\mu_{C(X)}(C(X))\right\}^{w_{1}}\left\{1-\mu_{\delta(X)}(\delta(X))\right\}^{w_{2}}\right]}$
Minimize $\frac{w_{1} v_{C(X)}(C(X))+w_{2} v_{\delta(X)}(\delta(X))-0.5\left[\left\{v_{C(X)}(C(X))\right\}^{w_{1}}\left\{v_{\delta(X)}(\delta(X))\right\}^{w_{2}}\right]}{1+0.5\left[\left\{v_{C(X)}(C(X))\right\}^{w_{1}}\left\{v_{\delta(X)}(\delta(X))\right\}^{w_{2}}\right]}$

subject to the same constraint as (6.ii)

\section{NUMERICAL ILLUSTRATION}

A welded beam (Ragsdell and Philips 1976,Fig. 2) has to be designed at minimum cost whose constraints are shear stress in weld $(\tau)$, bending stress in the beam $(\sigma)$ ,buckling load on the bar $(P)$, and deflection of the beam $(\delta)$. The design variables are $\left[\begin{array}{l}x_{1} \\ x_{2} \\ x_{3} \\ x_{4}\end{array}\right]=\left[\begin{array}{l}h \\ l \\ t \\ b\end{array}\right]$ where $h$ is the the weld size, $l$ is the length of the weld, $t$ is the depth of the welded beam, $b$ is the width of the welded beam.

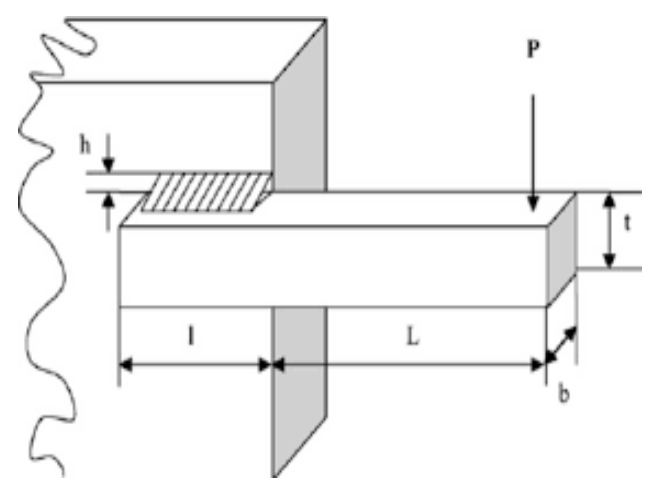

Fig.2. Design of the welded beam

\section{Cost Function}

The performance index appropriate to this design is the cost of weld assembly. The major cost components of such an assembly are (i) set up labour cost, (ii) welding labour cost, (iii) material cost.

$C(X) \equiv C_{0}+C_{1}+C_{2}$ where, $f(X)=$ cost function; $C_{0}=$ set up cost; $C_{1}=$ welding labour cost; $C_{2}=$ material cost;

Set up cost $C_{0}$ : The company has chosen to make this component a weldment, because of the existence of a welding assembly line. Furthermore, assume that 
fixtures for set up and holding of the bar during welding are readily available. The cost $C_{0}$ can therefore be ignored in this particular total cost model.

Welding labour cost $C_{1}$ : Assume that the welding will be done by machine at a total cost of $\$ 10 / \mathrm{hr}$ (including operating and maintenance expense). Furthermore suppose that the machine can lay down a cubic inch of weld in $6 \mathrm{~min}$. The labour cost is then

$$
C_{1}=\left(10 \frac{\$}{\mathrm{hr}}\right)\left(\frac{1}{60} \frac{\$}{\min }\right)\left(6 \frac{\mathrm{min}}{\mathrm{in}^{3}}\right) V_{w}=1\left(\frac{\$}{\mathrm{in}^{3}}\right) V_{w} . \text { Where } V_{w}=\text { weld volume, } \mathrm{in}^{3}
$$

Material cost $C_{2}: C_{2}=C_{3} V_{w}+C_{4} V_{B}$.Where $C_{3}=$ cost per volume per weld material. $\$ / \mathrm{in}^{3}=(0.37)(0.283) \quad ; \quad C_{4}=$ cost per volume of bar stock. $\$ / \mathrm{in}^{3}$ $=(0.37)(0.283) ; V_{B}=$ volume of bar,in ${ }^{3}$.From geometry $V_{w}=h^{2} l$; volume of the weld material $\left(\mathrm{in}^{3}\right) \quad V_{\text {weld }}=x_{1}^{2} x_{2}$ and $V_{B}=t b(L+l)$; volume of bar $\left(\mathrm{in}^{3}\right)$ $V_{b a r}=x_{3} x_{4}\left(L+x_{2}\right)$ Therefore cost function become $C(X)=h^{2} l+C_{3} h^{2} l+C_{4} t b(L+l)=1.10471 x_{1}^{2} x_{2}+0.04811 x_{3} x_{4}\left(14.0+x_{2}\right)$

\section{Engineering Relationship}

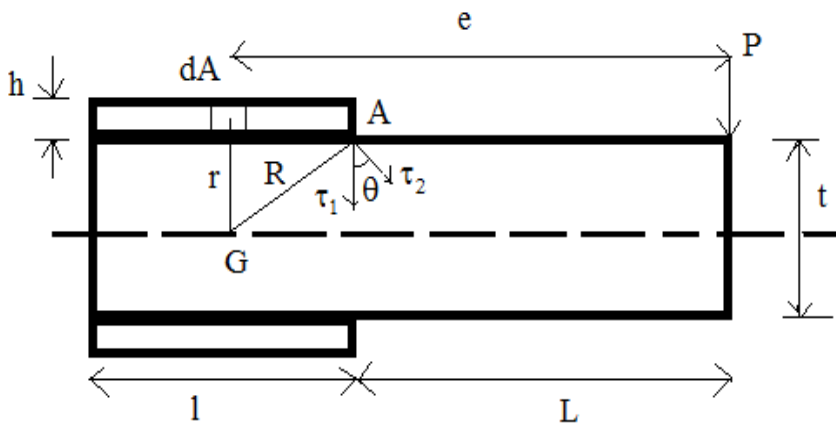

Fig 3. Shear stresses in the weld group.

\section{Maximum shear stress in weld group:}

To complete the model it is necessary to define important stress states

Direct or primary shear stress $\tau_{1}=\frac{\text { Load }}{\text { Throat area }}=\frac{P}{A}=\frac{P}{\sqrt{2} h l}=\frac{P}{\sqrt{2} x_{1} x_{2}}$

Since the shear stress produced due to turning moment $M=P . e$ at any section is proportional to its radial distance from centre of gravity of the joint ' $G$ ', therefore stress due to $M$ is proportional to $R$ and is in a direction at right angles to $R$. In other words $\frac{\tau_{2}}{R}=\frac{\tau}{r}=$ constant. Therefore $R=\sqrt{\left(\frac{l}{2}\right)^{2}+\left(\frac{h+t}{2}\right)^{2}}=\sqrt{\frac{x_{2}^{2}}{4}+\frac{\left(x_{1}+x_{3}\right)^{2}}{4}}$ 
Where, $\tau_{2}$ is the shear stress at the maximum distance $R$ and $\tau$ is the shear stress at any distance $r$. Consider a small section of the weld having area $d A$ at a distance $r$ from ' $G$ '. Therefore shear force on this small section $=\tau \times d A$ and turning moment of the shear force about centre of gravity $d M=\tau \times d A \times r=\frac{\tau_{2}}{R} \times d A \times r^{2}$.Therefore total turning moment over the whole weld area $M=\frac{\tau_{2}}{R} \int d A \times r^{2}=\frac{\tau_{2}}{R} J$. where $J=$ polar moment of inertia of the weld group about centre of gravity. Therefore shear stress due to the turning moment i.e. secondary shear stress, $\tau_{2}=\frac{M R}{\mathrm{~J}}$. In order to find the resultant stress, the primary and secondary shear stresses are combined vectorially. Therefore the maximum resultant shear stress that will be produced at the weld group, $\tau=\sqrt{\tau_{1}^{2}+\tau_{2}^{2}+2 \tau_{1} \tau_{2} \cos \theta}$, where, $\theta=$ Angle between $\tau_{1}$ and $\tau_{2}$. As $\cos \theta=\frac{l / 2}{R}=\frac{x_{2}}{2 R} ; \tau=\sqrt{\tau_{1}^{2}+\tau_{2}^{2}+2 \tau_{1} \tau_{2} \frac{x_{2}}{2 R}}$.

Now the polar moment of inertia of the throat area $(A)$ about the centre of gravity is obtained by parallel axis theorem, $J=2\left[I_{x x}+A+x^{2}\right]=2\left[\frac{A \times l^{2}}{12}+A \times x^{2}\right]=2 A\left(\frac{l^{2}}{12}+x^{2}\right)=2\left\{\sqrt{2} x_{1} x_{2}\left[\frac{x_{2}^{2}}{12}+\frac{\left(x_{1}+x_{3}\right)^{2}}{2}\right]\right\}$

Where, $A=$ throat area $=\sqrt{2} x_{1} x_{2}, l=$ Length of the weld, $x=$ Perpendicular distance between two parallel axes $=\frac{t}{2}+\frac{h}{2}=\frac{x_{1}+x_{3}}{2}$.

\section{Maximum bending stress in beam:}

Now Maximum bending moment $=P L$, Maximum bending stress $=\frac{T}{Z}$, where $T=P L$

$Z=$ section modulus $=\frac{I}{y} ; I=$ moment of inertia $=\frac{b t^{3}}{12} ; y=$ distance of extreme fibre from centre of gravity of cross section $=\frac{t}{2} ;$ Therefore $Z=\frac{b t^{2}}{6}$. So bar bending stress $\sigma(x)=\frac{T}{Z}=\frac{6 P L}{b t^{2}}=\frac{6 P L}{x_{4} x_{3}^{2}}$. 


\section{Maximum deflection in beam:}

Maximum deflection at cantilever tip $=\frac{P L^{3}}{3 E I}=\frac{P L^{3}}{3 E \frac{b t^{3}}{12}}=\frac{4 P L^{3}}{E b t^{3}}$

\section{Buckling load of beam:}

buckling load can be approximated by $P_{C}(x)=\frac{4.013 \sqrt{E I C}}{l^{2}}\left(1-\frac{a}{l} \sqrt{\frac{E l}{C}}\right)$

where, $I=$ moment of inertia $=\frac{b t^{3}}{12} ;$ torsional rigidity $C=G J=\frac{1}{3} t b^{3} G ; l=L ; a=\frac{t}{2}$;

$$
=\frac{4.013 \sqrt{E \frac{t^{2} b^{6}}{36}}}{L^{2}}\left(1-\frac{t}{2 L} \sqrt{\frac{E}{4 G}}\right)=\frac{4.013 \sqrt{E G x_{3}^{6} x_{4}^{6} / 36}}{L^{2}}\left(1-\frac{x_{3}}{2 L} \sqrt{\frac{E}{4 G}}\right) ;
$$

The single-objective optimization problem can be stated as follows

Minimize $g(x) \equiv 1.10471 x_{1}^{2} x_{2}+0.04811\left(14+x_{2}\right) x_{3} x_{4}$

Minimize $\delta(x) \equiv \frac{4 P L^{3}}{E x_{4} x_{3}^{2}}$

Such that

$$
\begin{aligned}
& g_{1}(x) \equiv \tau(x)-\tau_{\max } \leq 0 ; \\
& g_{2}(x) \equiv \sigma(x)-\sigma_{\max } \leq 0 ; \\
& g_{3}(x) \equiv x_{1}-x_{4} \leq 0 ; \\
& g_{4}(x) \equiv 0.10471 x_{1}^{2} x_{2}+0.04811 x_{3} x_{4}\left(14+x_{2}\right)-5 \leq 0 ; \\
& g_{5}(x) \equiv 0.125-x_{1} \leq 0 ; \\
& g_{6}(x) \equiv \delta(x)-\delta_{\max } \leq 0 ; \\
& g_{7}(x) \equiv P-P_{C}(x) \leq 0 ; \\
& 0.1 \leq x_{1}, x_{4} \leq 2.0 \\
& 0.1 \leq x_{2}, x_{3} \leq 2.0
\end{aligned}
$$


where $\quad \tau(x)=\sqrt{\tau_{1}^{2}+2 \tau_{1} \tau_{2} \frac{x_{2}}{2 R}+\tau_{2}^{2}} \quad ; \quad \tau_{1}=\frac{P}{\sqrt{2} x_{1} x_{2}} ; \tau_{2}=\frac{M R}{J} ; M=P\left(L+\frac{x_{2}}{2}\right)$; $R=\sqrt{\frac{x_{2}^{2}}{4}+\left(\frac{x_{1}+x_{3}}{2}\right)^{2}} ; J=\left\{\frac{x_{1} x_{2}}{\sqrt{2}}\left[\frac{x_{2}^{2}}{12}+\left(\frac{x_{1}+x_{3}}{2}\right)^{2}\right]\right\} ; \sigma(x)=\frac{6 P L}{x_{4} x_{3}^{2}} ; \delta(x)=\frac{4 P L^{3}}{E x_{4} x_{3}^{2}} ;$ $P_{C}(x)=\frac{4.013 \sqrt{E G x_{3}^{6} x_{4}^{6} / 36}}{L^{2}}\left(1-\frac{x_{3}}{2 L} \sqrt{\frac{E}{4 G}}\right) ; \quad P=$ Force on beam ; $L=$ Beam length beyond weld; $x_{1}=$ Height of the welded beam; $x_{2}=$ Length of the welded beam; $x_{3}=$ Depth of the welded beam; $x_{4}=$ Width of the welded beam; $\tau(x)=$ Design shear stress; $\sigma(x)=$ Design normal stress for beam material; $M=$ Moment of $P$ about the centre of gravity of the weld,$J=$ Polar moment of inertia of weld group; $G=$ Shearing modulus of Beam Material; $E=$ Young modulus; $\tau_{\max }=$ Design Stress of the weld; $\sigma_{\max }=$ Design normal stress for the beam material; $\delta_{\max }=$ Maximum deflection; $\tau_{1}=$ Primary stress on weld throat. $\tau_{2}=$ Secondary torsional stress on weld. Input data are given in table 1.

Table 1: Input data for crisp model (6)

\begin{tabular}{|c|c|c|c|c|c|}
\hline $\begin{array}{c}\text { Applied } \\
\text { load } P \\
(l b)\end{array}$ & $\begin{array}{l}\text { Beam } \\
\text { length } \\
\text { beyond } \\
\text { weld } L \\
\quad \text { (in) }\end{array}$ & $\begin{array}{c}\text { Young } \\
\text { Modulus } \\
\text { E } \\
(p s i)\end{array}$ & $\begin{array}{c}\text { Value of } \\
\qquad G \\
(p s i)\end{array}$ & $\begin{array}{l}\text { Maximum } \\
\text { allowable } \\
\text { shear } \\
\text { stress } \tau_{\max } \\
\quad(p s i)\end{array}$ & $\begin{array}{c}\text { Maximum } \\
\text { allowable } \\
\text { normal stress } \\
\sigma_{\max } \\
(p s i)\end{array}$ \\
\hline 6000 & 14 & $3 \times 10^{6}$ & $12 \times 10^{6}$ & $\begin{array}{l}13600 \\
\text { with fuzzy } \\
\text { region } \\
50\end{array}$ & $\begin{array}{c}30000 \\
\text { with fuzzy } \\
\text { region } \\
50\end{array}$ \\
\hline
\end{tabular}

Solution: : According to step 2 of 4.1.1, pay-off matrix is formulated as follows

$$
\begin{aligned}
& C(X) \quad \delta(X) \\
& X^{1}\left[\begin{array}{cc}
7.700387 & 0.2451363 \\
11.91672 & 0.1372000
\end{array}\right] \text {. }
\end{aligned}
$$

Here

$$
U_{C(X)}^{v}=U_{C(X)}^{\mu}=11.91672, L_{C(X)}^{\mu}=7.700387 L_{C(X)}^{v}=L_{C(X)}^{\mu}+\varepsilon_{C(X)}=7.700387+\varepsilon_{C(X)} \text {; }
$$

such that $0<\varepsilon_{C(X)}<(11.91672-7.700387)$; 
$U_{\delta(X)}^{\nu}=U_{\delta(X)}^{\mu}=0.2451363, L_{\delta(X)}^{\mu}=0.1372000$,

$L_{\delta(X)}^{v}=L_{\delta(X)}^{\mu}+\varepsilon_{\delta(X)}=0.1372000+\varepsilon_{\delta(X)} ;$

such that $0<\varepsilon_{\delta(X)}<(0.2451363-0.1372000)$

Here membership and non-membership function for objective functions $C(X), \delta(X)$ for $T=2$ are defined as follows

$\mu_{C(X)}(C(X))=\left\{\begin{array}{cc}1 & \text { if } C(X) \leq 7.700387 \\ \frac{e^{-2\left(\frac{C(X)-7.700387}{4.216333}\right)}-e^{-2}}{1-e^{-2}} & \text { if } 7.700387 \leq C(X) \leq 11.91672 \\ 0 & \text { if } C(X) \geq 11.91672\end{array}\right.$

$v_{C(X)}(C(X))=\left\{\begin{array}{cc}0 & \text { if } C(X) \leq 7.700387+\varepsilon_{C(X)} \\ \left(\frac{C(X)-\left(7.700387+\varepsilon_{C(X)}\right)}{4.216333-\varepsilon_{C(X)}}\right)^{2} & \text { if } 7.700387+\varepsilon_{C(X)} \leq C(X) \leq 11.91672 \\ 1 & \text { if } C(X) \geq 11.91672\end{array}\right.$

$\mu_{\delta(X)}(\delta(X))=\left\{\begin{array}{cc}1 & \text { if } \delta(X) \leq 0.1372000 \\ \frac{e^{-2\left(\frac{\delta(X)-0.1372000}{0.1079363}\right)}-e^{-2}}{1-e^{-2}} & \text { if } 0.1372000 \leq \delta(X) \leq 0.2451363 \\ 0 & \text { if } \delta(X) \geq 0.2451363\end{array}\right.$

$v_{\delta(X)}(\delta(X))=\left\{\begin{array}{cl}0 & \text { if } \delta(X) \leq 0.1372000+\varepsilon_{\delta(X)} \\ \left(\frac{\delta(X)-\left(0.1372000+\varepsilon_{\delta(X)}\right)}{0.1079363-\varepsilon_{\delta(X)}}\right)^{2} & \text { if } 0.1372000+\varepsilon_{\delta(X)} \leq \delta(X) \leq 0.2451363 \\ 1 & \text { if } \delta(X) \geq 0.2451363\end{array}\right.$ 
The optimal results of model (4) using t-norms and t-conorms are shown in table 2 to 4

Table 2. Optimal weight for equal importance on structural weight and deflection i.e $w_{1}=w_{2}=1 / 2$ and for $\varepsilon_{C(X)}=0.42 \varepsilon_{\delta(X)}=0.01$

\begin{tabular}{|c|c|c|c|c|c|c|}
\hline $\begin{array}{c}\text { Weighted } \\
\text { paremeterized } \\
\text { t-norm,t- } \\
\text { conorm } \\
\text { operator }\end{array}$ & $x_{1}($ in $)$ & $x_{2}($ in $)$ & $x_{3}($ in $)$ & $x_{4}($ in $)$ & $C(X)(\$)$ & $\delta(X)($ in $)$ \\
\hline $\begin{array}{c}\text { Min-max } \\
\text { operator }\end{array}$ & 1.112361 & 2 & 0.2304963 & 0.1 & 2.751561 & 0.2451363 \\
\hline Probabilistic & 0.125 & 2 & 0.1937823 & 0.125 & 5 & 0.2451363 \\
\hline Lukasewicz & 0.125 & 2 & 0.2305172 & 0.1 & 7.700387 & 0.1372 \\
\hline $\begin{array}{c}\text { Yager } \\
(1980)\end{array}$ & 0.1080241 & 2 & 0.1 & 0.1 & 0.03409746 & 0.2451350 \\
\hline $\begin{array}{c}\text { Hamacher } \\
(1978)\end{array}$ & 0.125 & 2 & 0.1 & 0.125 & 5 & 17561.60 \\
\hline
\end{tabular}

For equal importance, the extension of weighted yager-t-norm t-co-norm operator gives minimum cost of welding and probabilistic Hamacher give maximum deflection

Table 3. Optimal weight for more importance on structural weight $w_{1}=0.8, w_{2}=0.2$ and for $\varepsilon_{C(X)}=0.42 \varepsilon_{\delta(X)}=0.01$

\begin{tabular}{|c|c|c|c|c|c|c|}
\hline $\begin{array}{c}\text { Weighted } \\
\text { paremeterized } \\
\text { t-norm,t- } \\
\text { conorm } \\
\text { operator }\end{array}$ & $x_{1}($ in $)$ & $x_{2}($ in $)$ & $x_{3}($ in $)$ & $x_{4}($ in $)$ & $C(X)(\$)$ & $\delta(X)($ in $)$ \\
\hline $\begin{array}{c}\text { Min-max } \\
\text { operator }\end{array}$ & 1.321460 & 2 & 0.2304819 & 0.1 & 3.875958 & 0.2451363 \\
\hline $\begin{array}{c}\text { Probabilisti } \\
\text { c }\end{array}$ & 0.125 & 2 & 0.1937823 & 0.125 & 5 & 0.2451363 \\
\hline $\begin{array}{c}\text { Lukasewicz } \\
\text { Yager } \\
(1980)\end{array}$ & 0.125 & 2 & 0.1937823 & 0.125 & 5 & 0.1372 \\
\hline $\begin{array}{c}\text { Hamacher } \\
(1978)\end{array}$ & 1.286139 & 2 & 0.1 & 1.286139 & 3.753725 & 1706.813 \\
\hline
\end{tabular}

For more importance on welding cost ,the extension of weighted Hamacher-t-norm tco-norm operator gives minimum cost of welding and maximum deflection 
Table 4. Optimal weight for more importance on deflection $w_{1}=0.2, w_{2}=0.8$, and for

$$
\varepsilon_{C(X)}=0.42 \quad \varepsilon_{\delta(X)}=0.01
$$

\begin{tabular}{|c|c|c|c|c|c|c|}
\hline $\begin{array}{c}\text { Weighted } \\
\text { paremeterized } \\
\text { t-norm,t- } \\
\text { conorm } \\
\text { operator }\end{array}$ & $x_{1}($ in $)$ & $x_{2}($ in $)$ & $x_{3}($ in $)$ & $x_{4}($ in $)$ & $C(X)(\$)$ & $\delta(X)($ in $)$ \\
\hline $\begin{array}{c}\text { Min-max } \\
\text { operator }\end{array}$ & 0.125 & 2 & 0.2304049 & 0.1 & 0.5225783 & 0.2451363 \\
\hline $\begin{array}{c}\text { Probabilisti } \\
\text { c }\end{array}$ & 0.125 & 2 & 0.1937823 & 0.125 & 5 & 0.2451363 \\
\hline \begin{tabular}{c} 
Lukasewicz \\
\hline $\begin{array}{c}\text { Yager } \\
(1980)\end{array}$
\end{tabular} & 0.125 & 2 & 0.2305172 & 0.1 & 7.700 & 0.1372 \\
\hline $\begin{array}{c}\text { Hamacher } \\
(1978)\end{array}$ & 0.125 & 2 & 0.1937823 & 0.125 & 5 & 0.2451363 \\
\hline
\end{tabular}

For more importance on deflection ,the extension of weighted yager t-norm t-conorm operator gives minimum cost of welding where as weighted Lukasewicz t-norm t-co-norm operator gives minimum deflection .

\section{CONCLUSIONS}

In this paper, we have proposed a method to solve multi-objective structural model in intuitionistic fuzzy environment. Here binary t-norms are expressed in extended n-ary t-norms and discussed their basic properties and some special cases. The said model is solved by using t-norms and t-conorm based on intuitionistic fuzzy optimization technique. A main advantage of the proposed method is that it allows the user to concentrate on the actual limitations in a problem during the specification of the flexible objectives. This approximation method can be applied to optimize different models in various fields of engineering and sciences.

\section{ACKNOWLEDGEMENT:}

The research work of MridulaSarkar is financed by Rajiv Gandhi National Fellowship (F1-17.1/2013-14-SC-wes-42549/(SA-III/Website)),Govt of India.

\section{REFERENCES}


[1] K.M. Ragsdell, D.T. Phillips, Optimal design of a class of welded structures using geometric programming, ASME Journal of Engineering for Industries 98 (3)1976. 1021-1025, Series B.

[2] K. Deb, Optimal design of a welded beam via genetic algorithms, AIAA Journal 29 ,(11) 1991. 2013-2015.

[3] Deb, K., Pratap, A. and Moitra, S., Mechanical component design for multiple objectives using elitist non-dominated sorting GA. In Proceedings of the Parallel Problem Solving from Nature VI Conference,Paris, 16-20 September 2000, pp. 859-868.

[4] Coello, C.A.C. 2000b. Use of a self-adaptive penalty approach for engineering optimization problems. Comput. Ind., 41: 113-127. DOI: 10.1016/S0166-3615(99)00046-9.

[5] Carlos A. Coello Coello, 'Solving Engineering Optimization Problems with the Simple Constrained Particle Swarm Optimizer', Informatica 32 (2008) 319-326.

[6] Reddy , M. J.; Kumar, D. N.; ,’'An efficient multi-objective optimization algorithm based on swarm intelligence for engineering design" Engineering Optimization, Vol. 39, No. 1, January 2007, 49-68.

[7] Lee,K.S., Geem,Z.W. '”A new meta-heuristic algorithm for continuous engineering optimization: harmony search theory and practice' Comput. Methods Appl. Mech. Engrg. 194 (2005) 3902-3933.

[8] S. Kazemzadeh Azada, ,O. Hasançebia and O. K. Erol 'Evaluating efficiency of big-bang big-crunch algorithm in benchmark engineering optimization problems",,Int. J. Optim. Civil Eng., 2011; 3:495-505.

[9] Shuang Li,G. and Au,S.K. 'Solving constrained optimization problems via Subset Simulation'"2010 4th International Workshop on Reliable Engineering Computing (REC 2010),doi:10.3850/978-981-08-5118-7 069.

[10] Mahdavi , M. , Fesanghary , M., Damangir, E. , 'An improved harmony search algorithm for solving optimization problems" Applied Mathematics and Computation 188 (2007) 1567-1579.

[11] L. A. Zadeh, Fuzzy set, Information and Control, vol.8, no.3, pp.338-353, 1965.

[12] K. Atanassov, Intuitionistic fuzzy sets, Fuzzy sets and Systems, 20,87-96, 1986. 
[13] Dey.S,Roy.T.K.'Structural Design Optimization using basic T-Norm and TConorm based Intuitionistic Fuzzy Optimization Technique.’I.J.of Advanced Research in Computer Engineering and Technology,6(3),320327,2017 .

[14] Alsina, C., Trillas, E. and Valverde, I. "On some logical connectives for fuzzy set Theory”.J.Math.Anal.Appl.93,15-26,1981.

[15] G.Deschrijver,C.Cornelis and E.E Kerre." On the representation of intuitionistic fuzzy t-norms and t-conorms".IEEE Trans .Fuzzy Syst,12,4561,2004. Xeshui.Xu.’Intuitionistic Fuzzy Aggregation Operator" IEEE Trans .Fuzzy Syst,15(6),1179-1187,2007. 
